OPEN ACCESS

Edited by:

Wuelton Monteiro,

Fundação de Medicina Tropical

Doutor Heitor Vieira Dourado (FMT-

HVD), Brazil

Reviewed by:

Marcus Vinícius Guimarães Lacerda,

Fundação de Medicina Tropical Doutor Heitor Vieira Dourado (FMT-

HVD), Brazil

Marco Aurelio Sartim,

Federal University of Amazonas, Brazi

*Correspondence:

Charles J. Gerardo gerar001@mc.duke.edu

Specialty section:

This article was submitted to Vaccines and Molecular

Therapeutics,

a section of the journal

Frontiers in Immunology

Received: 11 November 2020 Accepted: 26 February 2021

Published: 15 March 2021

Citation:

Gerardo CJ, Silvius E, Schobel S,

Eppensteiner JC, McGowan LM,

Elster EA, Kirk AD and Limkakeng AT

(2021) Association of a Network of Immunologic Response and Clinical

Features With the Functional

Recovery From Crotalinae

Snakebite Envenoming.

Front. Immunol. 12:628113.

doi: 10.3389/fimmu.2021.628113

\section{Association of a Network of Immunologic Response and Clinical Features With the Functional Recovery From Crotalinae Snakebite Envenoming}

\author{
Charles J. Gerardo ${ }^{1 *}$, Elizabeth Silvius ${ }^{2}$, Seth Schobel ${ }^{3}$, John C. Eppensteiner ${ }^{1}$, \\ Lauren M. McGowan ${ }^{1}$, Eric A. Elster ${ }^{3}$, Allan D. Kirk ${ }^{1}$ and Alexander T. Limkakeng ${ }^{1}$ \\ ${ }^{1}$ Department of Surgery, Duke University, Durham, NC, United States, ${ }^{2}$ DecisionQ, Arlington, VA, United States, \\ ${ }^{3}$ Department of Surgery, Uniformed Services University of the Health Sciences, Bethesda, MD, United States
}

Background: The immunologic pathways activated during snakebite envenoming (SBE) are poorly described, and their association with recovery is unclear. The immunologic response in SBE could inform a prognostic model to predict recovery. The purpose of this study was to develop pre- and post-antivenom prognostic models comprised of clinical features and immunologic cytokine data that are associated with recovery from SBE.

Materials and Methods: We performed a prospective cohort study in an academic medical center emergency department. We enrolled consecutive patients with Crotalinae SBE and obtained serum samples based on previously described criteria for the Surgical Critical Care Initiative (SC2i)(ClinicalTrials.gov Identifier: NCT02182180). We assessed a standard set of clinical variables and measured 35 unique cytokines using Luminex Cytokine 35-Plex Human Panel pre- and post-antivenom administration. The PatientSpecific Functional Scale (PSFS), a well-validated patient-reported outcome of functional recovery, was assessed at $0,7,14,21$ and 28 days and the area under the patient curve (PSFS AUPC) determined. We performed Bayesian Belief Network (BBN) modeling to represent relationships with a diagram composed of nodes and arcs. Each node represents a cytokine or clinical feature and each arc represents a joint-probability distribution (JPD).

Results: Twenty-eight SBE patients were enrolled. Preliminary results from 24 patients with clinical data, 9 patients with pre-antivenom and 11 patients with post-antivenom cytokine data are presented. The group was mostly female (82\%) with a mean age of 38.1 $(S D \pm 9.8)$ years. In the pre-antivenom model, the variables most closely associated with the PSFS AUPC are predominantly clinical features. In the post-antivenom model, cytokines are more fully incorporated into the model. The variables most closely associated with the PSFS AUPC are age, antihistamines, white blood cell count (WBC), 
HGF, CCL5 and VEGF. The most influential variables are age, antihistamines and EGF. Both the pre- and post-antivenom models perform well with AUCs of 0.87 and 0.90 respectively.

Discussion: Pre- and post-antivenom networks of cytokines and clinical features were associated with functional recovery measured by the PSFS AUPC over 28 days. With additional data, we can identify prognostic models using immunologic and clinical variables to predict recovery from SBE.

Keywords: snake bite, antivenin, cytokine, prognostic model, predictive modeling, chemokine, Patient Specific Functional Scale

\section{BACKGROUND}

Snakebite envenoming (SBE) is a neglected tropical disease of global significance that primarily impacts low resource areas in low and middle income countries (LMICs), and snake-specific antivenoms are the cornerstone of therapy (1-8). Snake antivenoms have been used for over 100 years and have well demonstrated efficacy, but there remain significant safety, cost, and access to treatment considerations that limit their consistent use $(5,9)$. Currently available United States (US) antivenoms have a remarkable safety profile as compared to previous SBE biologics (10-16). Globally the safety profile of antivenoms is more mixed, and the risk-benefit profile of their use varies (17-19). This creates a potential barrier to their use in non-life threatening SBE where administration will not impact mortality, but can impact recovery and potential permanent disability (20).

Despite positive cost-effectiveness assessments of snake antivenoms, the cost to individual SBE victims can be beyond the financial means of many of those affected (21-23). This creates another barrier to use if the need for treatment is unclear due to the uncertainty of the severity and anticipated clinical course of the SBE patient $(24,25)$. There is also little economic incentive to pharmaceutical companies to develop and overcome the regulatory hurdles in order to distribute antivenom to where it is most needed (22, 26-28). This fact, combined with the lack of healthcare infrastructure in these low resource areas with a high prevalence of SBE, results in a lack of a consistently replaceable antivenom supply $(28,29)$. The concern for wasting antivenom when not absolutely needed, is accompanied by the risk of undertreatment for patients that would otherwise benefit $(12,30,31)$. The described barriers to antivenom use are particularly important as treatment is time dependent with worse outcomes when care is delayed (32-38).

The clinical courses of SBE patients are highly variable, even within a single envenoming species, which further contributes to uncertainty regarding both the value of administering antivenom and how much is necessary for an individual patient (39). Unfortunately, there is no validated and reliable method of predicting severity or recovery in SBE (40). Historically clinicians have had to rely primarily on their own clinical experience. This judgement has been augmented by treatment guidelines and severity scales, but these have not been evaluated as to whether they improve clinical outcomes $(7,41-43)$. This has led to wide practice variation in when and how much antivenom is administered even within the US where safety and access to antivenom are much less of a concern (44). A prognostic tool that could predict the severity of SBE would be valuable in overcoming the stated barriers to antivenom use and allow for therapy tailored to the individual in order to prevent both under- and over-treatment. Hence, the need for novel prognostic strategies persists.

Snake venom is a complex mixture of enzymatic and nonenzymatic proteins and peptides. In the sub-family Crotalinae, the species Crotalus horridus and Agkistrodon contortrix are known to have a large proportion of their venom composed of secretory phospholipase A2 (sPLA2), snake venom metalloproteases (SVMPs), and serine protease toxins. These toxins are of particular biologic importance and contribute to the venom-induced tissue injury, coagulopathy, and systemic symptoms seen in these SBEs (45, 46). However, the host inflammatory response is felt to also play a significant role in the pathophysiology and clinical syndrome of SBE (47-50). Consequently, evaluating inflammatory markers such as serum cytokines and chemokines give us a potential technique to evaluate immunologic response in SBE and hold promise as a component of a comprehensive prognostic strategy (48). Advances in enzyme-linked immune-assays using fluorescent bead technology now allow for simpler, reliable and rapid determination of cytokine levels (51). Consequently, the role of cytokines in other disease states is being investigated in LMICs using this technology (52-54).

Clinical features alone have not shown adequate prognostic performance in SBE, despite having some predictive value (37, $38,40,55,56)$. Combining clinical features with immunologic markers has been used to prognosticate outcomes in other disease states (57-60). An improved understanding of the immunologic response combined with available clinical features in SBE could inform a prognostic model to predict severity and recovery in SBE. The purpose of this study was to develop pre- and post-antivenom prognostic models comprised of clinical features and immunologic cytokine and chemokine data administration that are associated with recovery from SBE.

\section{MATERIALS AND METHODS}

\section{Design}

We performed a prospective cohort study at a single tertiarycare academic emergency department (ED) in the southeastern US in 2017 and 2019. 


\section{Participants}

We enrolled consecutive patients with acute snakebite envenoming (SBE) based on the Surgical Critical Care Initiative-enVenomation Investigation Pilot to Expedite Recovery (SC2i - VIPER) based on the SC2i - Tissue and Data Acquisition Protocol (TDAP) (ClinicalTrials.gov Identifier: NCT02182180). As the parent protocol has been previously described, we provide additional methods specific to the SC2i-VIPER sub-study that are necessary for reproducibility (57). We enrolled patients age 27 - 80 years presenting within the first 24 hours with verified acute SBE. No venom levels were obtained for enrollment, as all local venomous snakes are of the sub-family Crotalinae. Local species include Agkistrodon contortrix (copperhead) and Crotalus horridus (timber rattlesnake). Regionally transferred patients were eligible with the addition of the following potential Crotalinae species: Crotalus ademanteus (eastern diamondback rattlesnake), Sistrurus miliaris (pygmy rattlesnake) and Agkistrodon piscivorus (cottonmouth snake). Species identification was not required for enrollment, however the majority of investigator-identified species were copperhead snakes which predominate throughout the region. Exclusion criteria were pregnancy, prisoners, or inability to obtain consent during their stay for any reason. Informed consent was provided by a patient or proxy and the Duke University Institutional Review Board approved the study.

\section{Clinical Data}

We assessed clinical variables as per SC2i protocol which includes static pre-existing variables such as sex, age, comorbidities and dynamic clinical variables such as laboratory results, medications, and vital signs. Clinical data were collected from patients and by review of the electronic health record using standard operating procedures.

\section{Biological Sample Collection and Processing}

Samples from SBE patients were collected as soon as possible following their enrollment, ideally before antivenom administration when possible, as well as after initial antivenom administration. Whole blood was collected and serum was isolated at the site for the SC2i Consortium Biorepository following consortium-wide standard operating procedures and Guidelines for Good Clinical Laboratory Practice (GGCLP), and then stored until use for these analyses. The methods of sample collection and preparation have been reported, but are briefly encapsulated here for clarity (61).

\section{Sample Preparation}

Frozen serum samples were thawed on wet ice for filtering. The samples could have a single freeze-thaw cycle. They were mixed and $400 \mathrm{uL}$ were transferred to a $1.5 \mathrm{~mL}$ tube and centrifuged at 12,000g for 15 minutes at 4 degrees Celsius. A 350uL sample was transferred into a $0.65 \mathrm{um}$ filter tube (Millipore, Billerica, MA). They were centrifuged $12,000 \mathrm{~g}$ for 30 minutes at 4 degrees Celsius. We then aliquoted $120 \mathrm{uL}$ of the filtered samples into clean tubes. We then flash froze samples in liquid nitrogen and stored in -80 degree Celsius freezer. The maximum time from freeze to thaw was less than one year.
After filtering, samples were thawed and a 1:50 dilution of samples were used. We measured levels of 35 unique cytokines using Luminex Cytokine 35-Plex Human Panel (Thermo Fisher Scientific, Waltham, MA) pre- and post-antivenom administration using standard cytokine preparation per kit instructions. A $1 \mathrm{x}$ wash preparation was created and used for all washes. Standard plates were prepared and 50ul of sample, standards, and incubation buffer were added to all wells and allowed to incubate for 2 hours. After washing, detection antibodies were added and incubated for one hour. After another wash, streptavidin-RPE was added and incubated for 30 minutes. These were washed again and beads were resuspended in wash buffer. For 35 plex analysis, we used Invitrogen Catalog\# LHC6005M kits are from Thermo Fisher Scientific. The Comprehensive Human Cytokine Magnetic 35Plex Panel provides reagents for the accurate, reproducible, and sensitive quantitation of human proteins including: EGF, Eotaxin, FGF-basic, G-CSF, GM-CSF, HGF, IFN-alpha, IFNgamma, IL-1 beta, IL-1 alpha, IL-1RA, IL-2, IL-2R, IL-3, IL-4, IL-5, IL-6, IL-7, IL-8, IL-9, IL-10, IL-12, IL-13, IL-15, IL-17A, IL17F, IL-22, CXCL10, CCL2, CXCL9, CCL3, CCL4, CCL5, TNFalpha, VEGF.

\section{Outcomes}

The Patient-Specific Functional Scale (PSFS), a well-validated patient-reported outcome of functional recovery, was assessed at $0,7,14,21$ and 28 days. Individual patient PSFS recovery curves over time were assessed to determine the PSFS area under the patient curve (AUPC) over the course of 28 days. The median PSFS AUPC was determined. Patients with PSFS AUPC great than or equal to the median were considered to have good recovery and those less than the median defined poor recovery.

\section{Data Analysis}

For this preliminary analysis, we report baseline characteristics of all patients with available data. Continuous variables are summarized using mean, medians and interquartile range (IQR) as appropriate for the distribution of data.

To account for the small sample size with high dimensionality of the data set, we performed Bayesian Belief Network (BBN) modeling using a machine learning technique to build directed acyclic graphs that represent relationships with a diagram composed of nodes and arcs. Each node represents a cytokine, chemokine or clinical feature and each arc represents a jointprobability distribution (JPD). The JPDs were determined in a step-wise fashion over multiple iterations. We report associations of this cytokine/chemokine/clinical feature model with the patient's recovery (PSFS AUPC). We built the BBNs employing FasterAnalytics Version 7.0. A BBN starts with an observation, then calculates the relationships of various variables that are likely to impact that observation based on known probabilities (beliefs). BBNs are very useful for illustrating the differences in the immune systems of different patient groups since they are represented by acyclic directed graphs. These graphs can be overlaid on each other so that we can easily see 
where there are differences in the cytokine, chemokine and clinical feature joint probability distributions. In this case, BBN's can 1) identify which relationships exist between variables 2) allow us to measure the magnitude of relationships (one variable on another) but also the number of relationships for any given variable 3) handle a large number of variables without large datasets 4) compare which relationships exist and how many relationships exist between the two groups of recovery (good and poor). These models also deal easily with missing data, which was anticipated in this cytokine data set.

For the Luminex 35-plex kit, we built four Bayesian Belief Networks $(\mathrm{BBN})$ for preliminary modeling using a Minimum Descriptive Lengths (MDL) of 0.01. The MDL measures the trade-off between goodness-of-fit and complexity of the model. Models with lower MDLs have more complexity and are more likely to be overfit. For each time point (pre- and postantivenom), we built one model on the full data set and then models using leave-one-out. For each timepoint, we looked at the models and found which variables were first-degree associates of recovery. We used these variables in final modeling. If a variable was missing for more than $20 \%$ of records, we dropped the variable for the models with that kit.

We used leave-one-out cross validation. We used the scores from each test case to build a receiver operating characteristic (ROC) and measure the area under the curve (AUC). We consider any AUC of 0.6 or above to be a good differentiator between good and poor recovery. We also provided statistics about the connectivity of the models.

\section{RESULTS}

\section{Demographics}

Twenty-eight snake envenoming patients were enrolled. Preliminary demographic data and PSFS AUPC is available for 24 patients with cytokine and chemokine data available from 9 pre-antivenom and 11 post-antivenom patients. The group was mostly female $(82 \%)$ with a mean age of 38.1 ( $\mathrm{SD} \pm 9.8$ ) years.

Table 1.
TABLE 1 | Demographics and clinical features of enrolled patients.

\begin{tabular}{lcc}
\hline & $\mathbf{N}=\mathbf{2 4}$ & $\mathbf{N}=\mathbf{1 1}$ \\
\hline Age -Min, Median, Max & $25,45,78$ & $27,35,56$ \\
Age - 1stQ, Mean, 3rdQ, & $33.5,46.2,56$ & $30.5,38.1,46.5$ \\
Sex (\% Female) & $17(70.1 \%)$ & $9(81.8 \%)$ \\
White & $22(91.6)$ & $10(90.1 \%)$ \\
Asian & $2(8.3 \%)$ & $1(9.1 \%)$ \\
Comorbidity (at least one) & $11(45.8 \%)$ & $5(54.5 \%)$ \\
Immunosuppressed & $1(4.1 \%)$ & 0
\end{tabular}

\section{Recovery}

The patients' functional recovery as measured by the PSFS AUPC, for the 24 patients with available data, had a median of 0.73 (range $0.43,0.94$ ) and is represented graphically in Figure 1.

\section{Pre- and Post-Antivenom Cytokines}

The pre- and post-antivenom cytokines and chemokines were compared in Figure 2. There were changes in log values pre- and post-antivenom with increases in the median of the log of basic fibroblast growth factor (FGFBasic)(1.74 to 2.97), interleukin-2 receptor (IL02R)(3.04 to 4.50) and regulated on activation, C-C motif ligand chemokine (CCL5)(0.28 to 0.41$)$ and a decrease in human growth factor (HGF)(6.24 to 5.85).

The pre-antivenom individual cytokines and chemokines values were compared between good and poor functional recovery and are represented in Figure 3. In the preantivenom data, patients with poor recovery have higher log values of the eosinophil chemokine subfamily EOTAXIN (poor recovery median 4.35, good recovery median 3.99), HGF (6.44, 5.59), interleukin-1 receptor antagonist (IL01Ra) (3.98, 3.96), IL10 (1.90, 1.43), IL12 (4.47, 4.14), and C-C motif ligand 2 (CCL2) $(6.06,5.69)$. Patient with poor recovery had lower log values of CXCL10: C-X-C motif ligand $10(1.39,1.74), \mathrm{C}-\mathrm{C}$ motif ligand 4 (CCL4) $(3.71,4.40)$, CCL5 $(7.26,7.33)$, and vascular endothelial growth factor (VEGF) $(0.26,0.92)$. The postantivenom individual cytokines and chemokines values were compared between good and poor functional recovery and are represented in Figure 4. Patients with poor recovery have higher values of endothelial growth factor (EGF) (poor recovery median
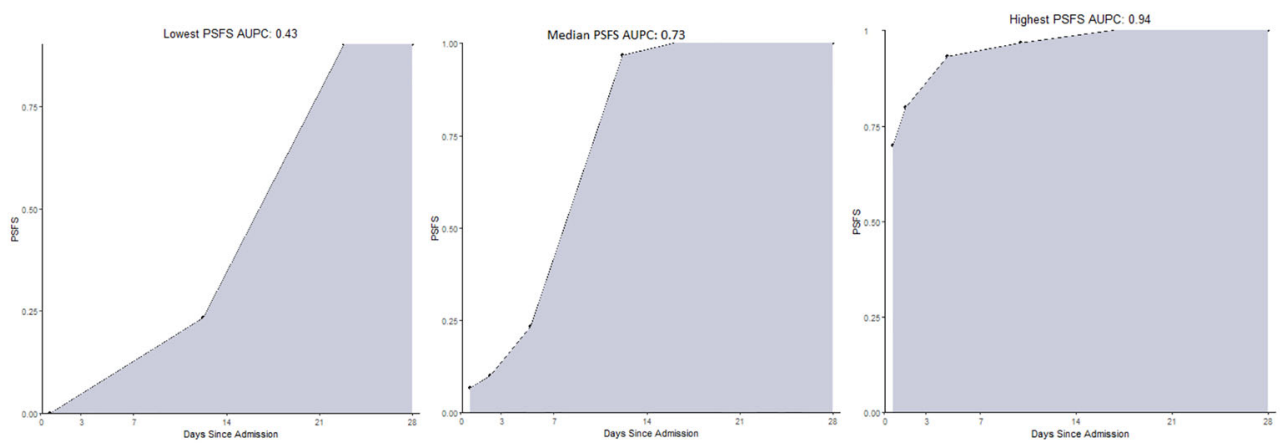

FIGURE 1 | The range of the Patient-Specific Functional Scale Area Under the Patient Curves at 28 days for 24 patients with available preliminary data. (examples lowest, median, highest). PSFS AUPC, Patient-Specific Functional Scale Area Under the Patient Curve. 


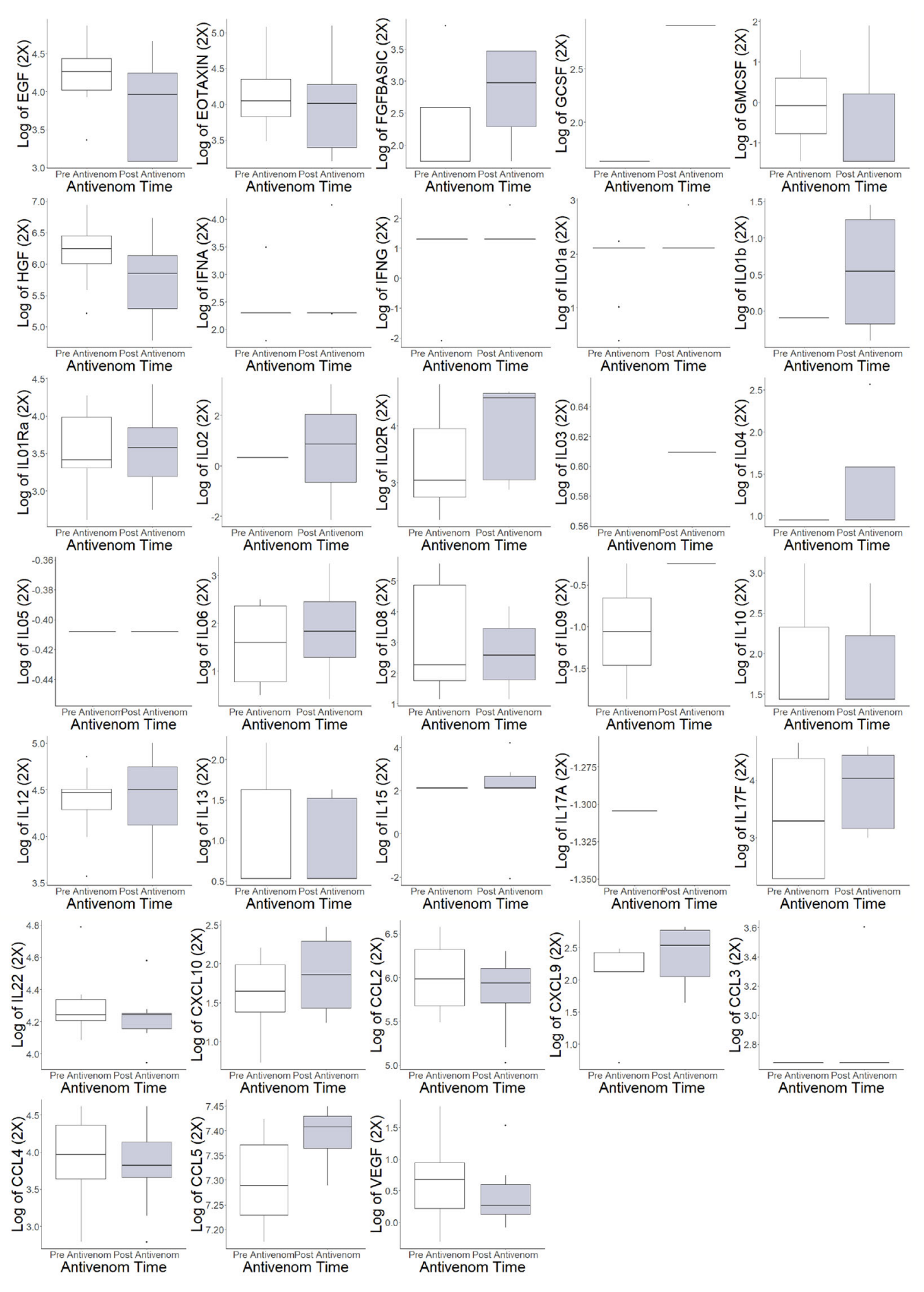

FIGURE 2 | Individual cytokine and chemokine values pre- and post-antivenom treatment. EGF: endothelial growth factor, EOTAXIN: eosinophil chemotactic proteins, "FGFBASIC: fibroblastic growth factor, basic, GCSF: granulocyte colony stimulating factor, GMCSF: ganulocyte-macrophage colony stimulating factor, *HGF: hepatocyte growth factor, IFNA: interferon alpha, IFNG: interferon gamma, IL01interleukin 1a, IL01b: interleukin 1b, IL01Ra: Interleukin 1 receptor antagonist, IL02: interleukin 2, ${ }^{*}$ ILO2R: interleukin 2 receptor, IL03: interleukin 3, IL04: interleukin 4, IL05: interleukin 5, IL06: interleukin 6, IL08: interleukin 8, IL09: interleukin 9, IL10: interleukin 10, IL12: interleukin 12, IL13: interleukin 13, IL15: interleukin 15, IL17A: interleukin 17A, IL17F: interleukin 17F, IL22: interleukin 22, CXCL10: C-X-C motif ligand 10, CCL2: C-C motif ligand 2, CXCL9: C-X-C motif ligand 9, CCL3: C-C motif ligand 3, CCL4: C-C motif ligand 4, ${ }^{*} C C L 5$ : C-C motif ligand 5, VEGF: vascular endothelial growth factor. *Difference in populations based on box plots. 
4.25, good recovery median 3.08), $\operatorname{HGF}(6.09,5.27)$, IL01Ra $(3.75,3.33)$, and IL12 $(4.54,4.34)$ with lower values of CXCL10 (1.62, 2.29), CCL2 (5.90, 6.05), and CCL4 (3.71, 3.98).

\section{Prognostic Modeling Networks}

In order to illustrate the effect antivenom has on the networks, the pre-and post-antivenom modeling networks are presented in Figures 5 and 6, respectively. In the pre-antivenom model, the variables most closely associated with the PSFS AUPC (i.e. recovery) are predominantly clinical features such as age, respiratory rate, $\mathrm{CO} 2$ (mostly bicarbonate) as measured by the basic metabolic panel, white blood cell count (WBC) and the need for antihistamines. These variables are also the most influential on the model, according to the Minimum Description Length (MDL) score. In the post-antivenom model, cytokines and chemokines are more fully incorporated into the model. The variables most closely associated with the PSFS AUPC are age, antihistamines, HGF, CCL5 and VEGF. The most influential variables are age, antihistamines and ECF. Within the limits of this small preliminary dataset, both the pre- and post-antivenom models perform well with AUCs of 0.87 and 0.90 respectively. Model stability was not assessed.

\section{DISCUSSION}

Using a preliminary data set from patients with SBE, we identified distinct pre- and post-antivenom patterns of clinical features combined with cytokines and chemokines that are associated with recovery. These findings are important as they demonstrate the feasibility of using this technique to develop a more predictive prognostic model than currently exists. Ultimately this could result in a clinical decision support tool capable of determining thresholds to treat with antivenom or other novel therapeutics and/or informing the need for escalating therapies. This individually tailored therapy approach holds the potential to most efficiently improve patient outcomes from SBE while preserving treatment for those likely to benefit.

The functional impact of SBE varies widely from patient to patient even within a given envenoming species. Specific SBErelated disabilities may impact different functions from work, to self-care, domestic life, social interactions, or civic life to name a few (62-64). By predicting and mitigating morbidity, we can maximize the economic, societal, and individual impact of SBE treatment. This is of particular importance in low-resourced areas of LMICs where the majority of SBE-associated negative consequences occur (5).

Our technique has already been used to enhance the care of other disease states such as infection and injury. In 2019, Gelbard et al. developed models using clinical data, cytokines, chemokines and growth factors that predicted severe sepsis and organ space infections following laparotomy for abdominal trauma (58). This modeling approach has expanded to combat trauma, where a predictive model composed of clinical features and immunologic biomarkers can accurately predict pneumonia in a predominantly blast-injured cohort of patients (59).
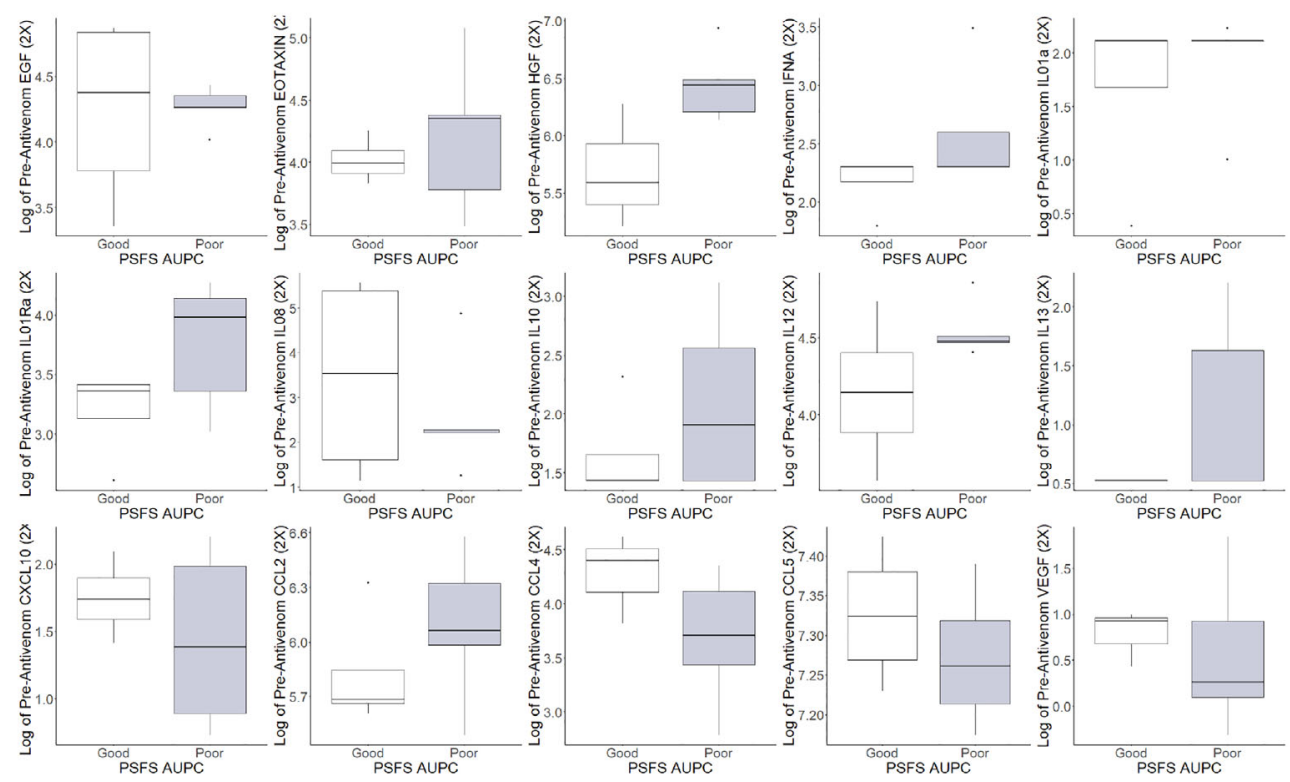

FIGURE 3 | Pre-antivenom cytokines and chemokines values of patients with good and poor recovery. EGF: endothelial growth factor, *EOTAXIN: eosinophil chemotactic proteins, *HGF: hepatocyte growth factor, IFNA: interferon alpha, IL01a: interleukin 1a, IL01Ra: Interleukin 1 receptor antagonist, IL08: interleukin 8, ${ }^{*}$ IL10: interleukin 10, ${ }^{*}$ LL12: interleukin 12, IL13: interleukin 13, ${ }^{*} \mathrm{CXCL10:} \mathrm{C}-\mathrm{X}-\mathrm{C}$ motif ligand 10, *CCL2: C-C motif ligand 2, ${ }^{*} \mathrm{CCL} 4:$ : $-\mathrm{C}$ motif ligand 4, ${ }^{*} \mathrm{CCL5}$ : C-C motif ligand 5, *VEGF: vascular endothelial growth factor. *Difference in populations based on box plots. 

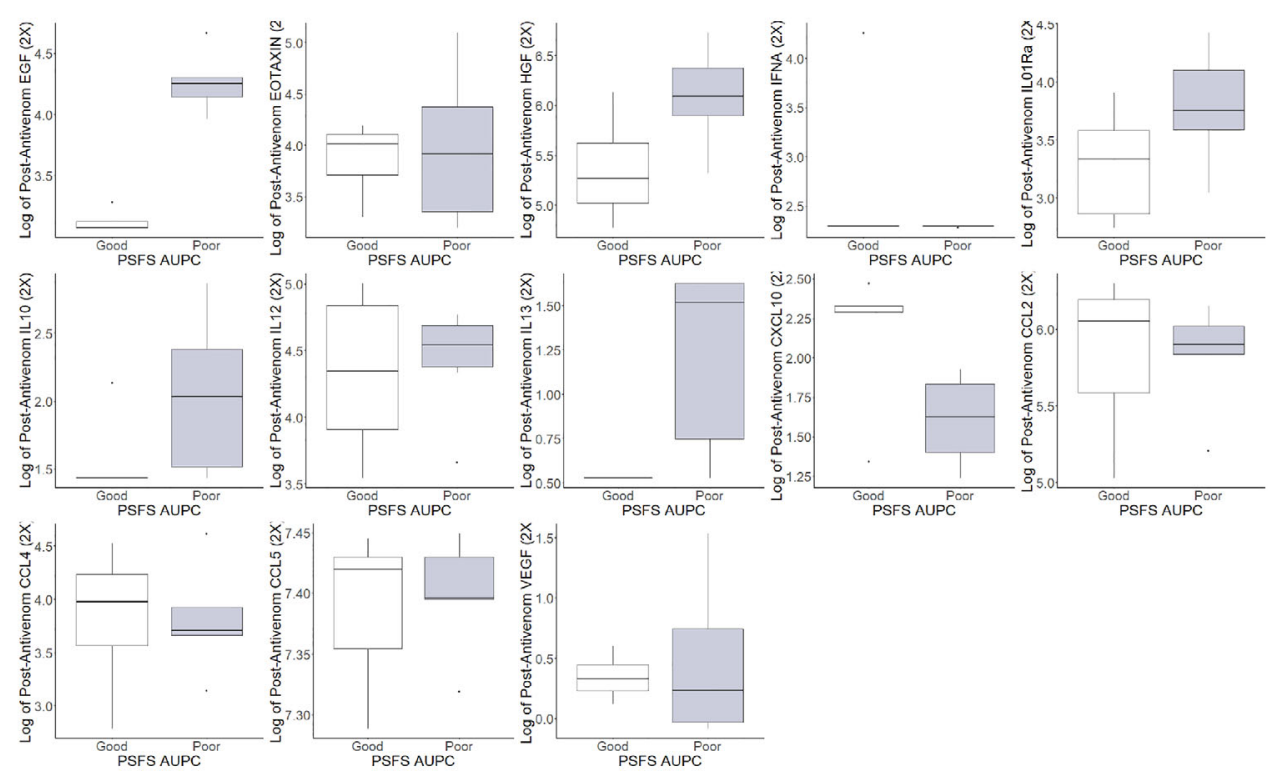

FIGURE 4 | Post-antivenom cytokine and chemokine values of patients with good and poor recovery. *EGF: endothelial growth factor, EOTAXIN: eosinophil chemotactic proteins, "HGF: hepatocyte growth factor, IFNA: interferon alpha, *IL01RA: Interleukin 1 receptor antagonist, IL10: interleukin 10, *IL12: interleukin 12, IL13: interleukin 13, *CXCL10: C-X-C motif ligand 10, ${ }^{*}$ CCL2: C-C motif ligand 2, *CCL4: C-C motif ligand 4, CCL5: C-C motif ligand 5, VEGF: vascular endothelial growth factor. *Difference in populations based on box plots.

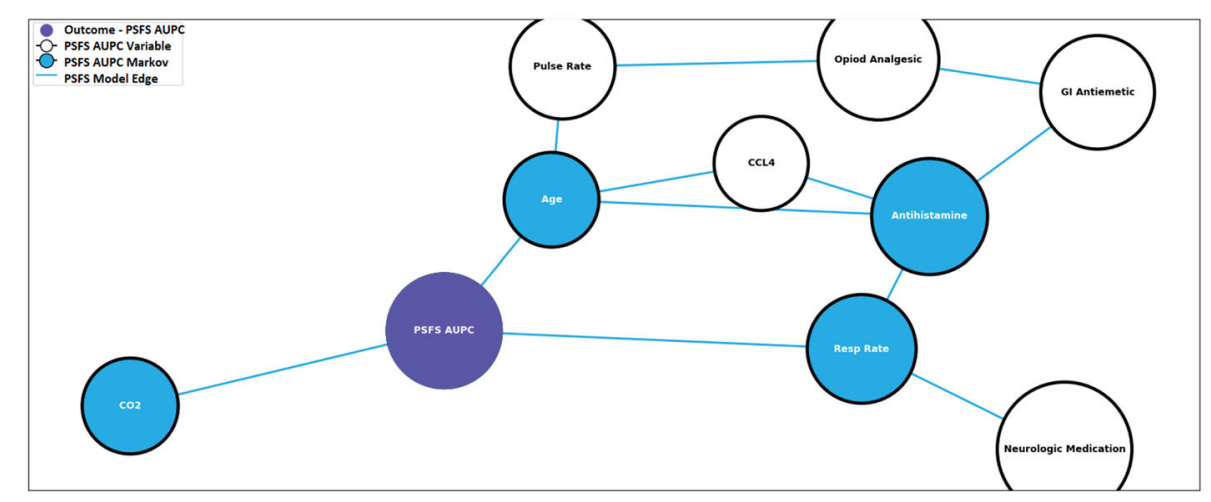

FIGURE 5 | Pre-antivenom prognostic model predictive of recovery. PSFS AUPC: Patient-Specific Functional Scale Area Under the Patient Curve, CCL4: C-C motif ligand 4, Gl: gastro-intestinal, $\mathrm{CO}_{2}$ : carbon dioxide, Resp Rate: respiratory rate.

By expanding this technique to SBE, we have built on the prior work evaluating both the clinical features predicting severity and studies of the association of soluble biomarkers with severity $(47,48,65,66)$. In doing so, we are able to overcome some of the limitations of the initial forays into prognostication in SBE. For example, a multitude of basic clinical features have been assessed in predicting severe SBE $(33,34,36,37,40,43$, $56,67,68)$. A number of these established clinical features, such as young age and time to care, are now known to increase the likelihood of a severe envenoming. However, none can adequately exclude severe SBE (40). Consequently, they have not been assessed as a tool to determine the need to treat or not to treat with antivenom. Additionally, the commonly applied laboratory testing have not even been demonstrated to confidently predict their own future trend $(40,56,69)$. Finally, combinations of these clinical features and laboratory tests have been combined into a number of grading scales $(42,43)$. However, none of these have had comprehensive psychometric evaluations that assess their validity and reliability in predicting patient-centered outcomes.

Similarly, the associations of soluble immunologic biomarkers such as cytokines, chemokines and complement with acute findings of tissue injury are now being studied. In 2019, Ibiapina and colleagues evaluated the relationships between 


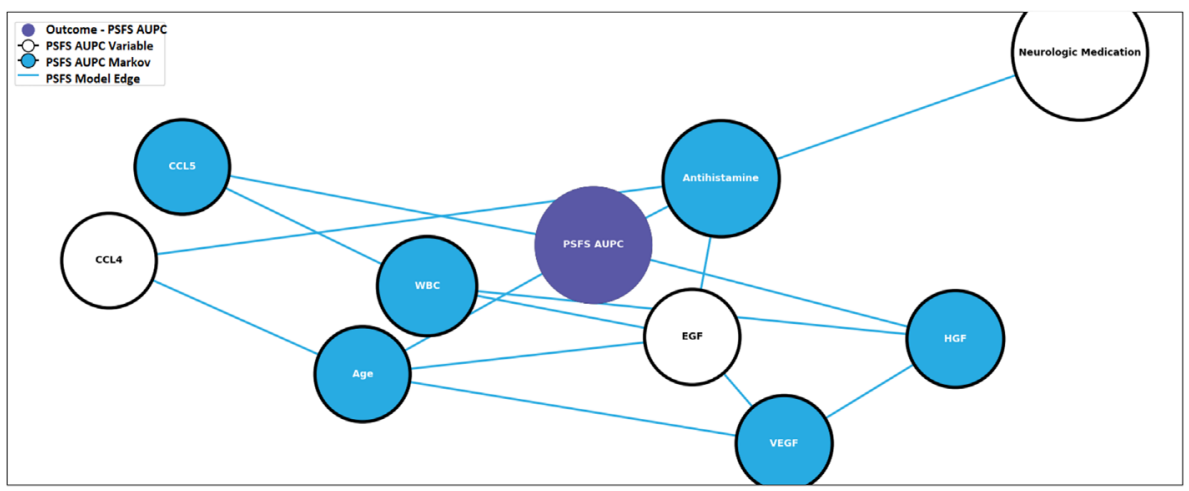

FIGURE 6 | Post-antivenom prognostic model predictive of recovery. PSFS AUPC: Patient-Specific Functional Scale Area Under the Patient Curve, CCL4: C-C motif ligand 4, CCL5: C-C motif ligand 5, ECG: endothelial growth factor, HGF: hepatocyte growth factor, VEGF: vascular endothelial growth factor, WBC: white blood cell count.

seventeen of these biomarkers and tissue injuries. Relationships were evaluated between biomarkers of healthy controls and patients with mild or severe tissue injury in Bothrops asper SBE. Important associations were established with individual biomarkers as well as classes of immune response polarizations such as Th-1,Th-2, and Th17. However, their results are limited by the lack of an established relationship between acute tissue findings they evaluated, such as swelling, blisters, or superficial necrosis, with important patient-centered outcomes such as functional recovery (48).

Our study also shows relationships with individual immunologic biomarkers and clinical outcomes. For example, Th-2 polarizationassociated cytokines such as IL-10 and IL-13 were increased in patients with poor outcomes, as were the chemokines CXCL 10, and the growth factors EGF and HGF. Our preliminary data is not yet robust enough to reliably characterize the comprehensive nature of the immune response in SBE. However, for prognostication purposes, it appears that we can overcome prior limitations by using advanced machine learning models using clinical and immunologic laboratory features to accurately predict a well-validated, patientcentered, functional outcome.

In addition to prognostic model development, our data give us further insight into potential immunologic therapeutic targets in the sterile inflammatory disease state of SBE. For example, nonsteroidal anti-inflammatory drugs have been typically avoided in SBE due to their antiplatelet effects and the potential hemotoxicity of snake venom. However, the study of their beneficial impact on cytokine response and wound healing in trauma demonstrates the need to further study NSAIDs and immune-mediated outcomes in an appropriate SBE population $(61,70,71)$. Ultimately, a comprehensive evaluation of individual biomarkers including their change over the natural history of $\mathrm{SBE}$, their relationship with outcome, the impact of antivenom on their levels, their role in prognostic modeling, and the presence of existing therapeutics targeting the specific biomarker should all impact their candidacy as a viable therapeutic target.

Moreover, consistency of results will play an important role in this work. Interestingly, this study did not find a significant elevation of tumor necrosis factor alpha (TNF-alpha). Previous work has shown a relationship between increasing TNF and SBE (72-74). Our unanticipated finding may be due to the predominant species enrolled in this study, Agkistrodon contortrix, is known to typically have a less severe clinical course. However, this finding should be verified using other cytokine assays to better characterize its role compared to SBE from other species.

\section{LIMITATIONS/FUTURE DIRECTIONS}

Our preliminary findings are limited by the small sample size currently available for analysis. This resulted in some atypical demographic patterns such a female predominant study population; whereas, a similar SBE population in our geographic region is typically slightly male predominant (75). This also limits our ability to perform more nuanced analysis such as evaluating outcomes based on the total amount of antivenom administered. Despite this, the models do perform well and the anticipated additional data can be used to refine the models and assess model stability. In order to establish prognostic models that would be usable in clinical practice the immunologic evaluation relies on cytokine and chemokine data that can be easily obtainable in a timely fashion. A more comprehensive immunologic evaluation such as complement levels, flow cytometry, transcriptomics, and venom associated molecular patterns (VAMPS) would give a more global picture of the immunologic response to SBE than is available in this preliminary analysis. Likewise additional clinical data such as presenting severity grade or time from bite to presentation could be incorporated into the model.

This study is limited to only a few species of Crotalinae snakes and the predominant species is known to have a less severe clinical course than others. Yet, our data demonstrate a wide distribution of recovery as measured by PSFS AUPC over 28 days. The heterogeneity of the disease itself and the utility of our chosen outcome measure allows us to discriminate between patient's recovery and develop robust prognostic models. This technique can be easily applied to other Crotalinae snake species. 
Lastly, as this is preliminary work, we have not used the models to determine thresholds to treat nor assessed clinical efficacy of implementing this application.

\section{CONCLUSION}

Pre- and post-antivenom networks of cytokines/chemokines and clinical features were associated with functional recovery measured by the PSFS AUPC over 28 days. With additional patient data, we can identify prognostic models using immunologic and clinical variables to predict recovery from snake envenoming.

\section{DATA AVAILABILITY STATEMENT}

The raw data supporting the conclusions of this article will be made available by the authors, without undue reservation.

\section{REFERENCES}

1. Minghui R, Malecela MN, Cooke E, Abela-Ridder B. WHO's Snakebite Envenoming Strategy for prevention and control. Lancet Glob Health (2019) 7(7):e837-8. doi: 10.1016/S2214-109X(19)30225-6

2. Brenes-Chacón H, Gutiérrez JM, Camacho-Badilla K, Soriano-Fallas A, Ulloa-Gutierrez R, Valverde-Muñoz K, et al. Snakebite envenoming in children: A neglected tropical disease in a Costa Rican pediatric tertiary care center. Acta Trop (2019) 200:105176. doi: 10.1016/j.actatropica.2019.105176

3. Pach S, Le Geyt J, Gutiérrez JM, Williams D, Maduwage KP, Habib AG, et al, et al. Paediatric snakebite envenoming: the world's most neglected "Neglected Tropical Disease"? Arch Dis Child (2020). doi: 10.1136/ archdischild-2020-319417

4. Kasturiratne A, Wickremasinghe AR, de Silva N, Gunawardena NK, Pathmeswaran A, Premaratna R, et al. The global burden of snakebite: a literature analysis and modelling based on regional estimates of envenoming and deaths. PloS Med (2008) 5(11):e218. doi: 10.1371/journal.pmed.0050218

5. Williams DJ, Faiz MA, Abela-Ridder B, Ainsworth S, Bulfone TC, Nickerson $\mathrm{AD}$, et al. Strategy for a globally coordinated response to a priority neglected tropical disease: Snakebite envenoming. PLoS Negl Trop Dis (2019) 13(2): e0007059. doi: 10.1371/journal.pntd.0007059

6. Chippaux JP. Snake-bites: appraisal of the global situation. Bull World Health Organ (1998) 76(5):515-24.

7. Lavonas EJ, Ruha A-M, Banner W, Bebarta V, Bernstein JN, Bush SP, et al. Unified treatment algorithm for the management of crotaline snakebite in the United States: results of an evidence-informed consensus workshop. BMC Emerg Med (2011) 11:2. doi: 10.1186/1471-227X-11-2

8. Harrison RA, Gutiérrez JM. Priority actions and progress to substantially and sustainably reduce the mortality, morbidity and socioeconomic burden of tropical snakebite. Toxins (Basel) (2016) 8(12). doi: 10.3390/toxins8120351

9. Pucca MB, Cerni FA, Janke R, Bermúdez-Méndez E, Ledsgaard L, Barbos JE, et al. History of envenoming therapy and current perspectives. Front Immunol (2019) 10:1598. doi: 10.3389/fimmu.2019.01598

10. Dart RC, Seifert SA, Boyer LV, Clark RF, Hall E, McKinney P, et al. A randomized multicenter trial of crotalinae polyvalent immune Fab (ovine) antivenom for the treatment for crotaline snakebite in the United States. Arch Intern Med (2001) 161(16):2030-6. doi: 10.1001/archinte.161.16.2030

11. Bush SP, Ruha A-M, Seifert SA, Morgan DL, Lewis BJ, Arnold TC, et al. Comparison of $\mathrm{F}\left(\mathrm{ab}{ }^{\prime}\right) 2$ versus $\mathrm{Fab}$ antivenom for pit viper envenomation: a prospective, blinded, multicenter, randomized clinical trial. Clin Toxicol (2015) 53(1):37-45. doi: 10.3109/15563650.2014.974263

\section{ETHICS STATEMENT}

The studies involving human participants were reviewed and approved by Duke University School of Medicine Institutional Review Board. The patients/participants provided their written informed consent to participate in this study.

\section{AUTHOR CONTRIBUTIONS}

All authors listed have made a substantial, direct, and intellectual contribution to the work and approved it for publication.

\section{FUNDING}

Grant funding for this research: United States Department of Defense. USUHS HT9404-13-1-0032 and USUHS HU001-152-0001.

12. Gerardo CJ, Quackenbush E, Lewis B, Rose SR, Greene S, Toschlog EA, et al. The Efficacy of Crotalidae Polyvalent Immune Fab (Ovine) Antivenom Versus Placebo Plus Optional Rescue Therapy on Recovery From Copperhead Snake Envenomation: A Randomized, Double-Blind, Placebo-Controlled, Clinical Trial. Ann Emerg Med (2017) 70(2):233-44.e3. doi: 10.1016/ j.annemergmed.2017.04.034

13. Dart RC, Seifert SA, Carroll L, Clark RF, Hall E, Boyer-Hassen LV, et al. Affinity-purified, mixed monospecific crotalid antivenom ovine Fab for the treatment of crotalid venom poisoning. Ann Emerg Med (1997) 30(1):33-9. doi: 10.1016/s0196-0644(97)70107-0

14. Mullins ME, Gerardo CJ, Bush SP, Rose SR, Greene S, Quackenbush EB, et al. Adverse Events in the Efficacy of Crotalidae Polyvalent Immune Fab Antivenom vs Placebo in Recovery from Copperhead Snakebite Trial. South Med J (2018) 111(12):716-20. doi: 10.14423/SMJ.0000000000000902

15. Kravitz J, Gerardo CJ. Copperhead snakebite treated with crotalidae polyvalent immune fab (ovine) antivenom in third trimester pregnancy. Clin Toxicol (2006) 44(3):353-4. doi: 10.1080/15563650600584725

16. Clark RF, McKinney PE, Chase PB, Walter FG. Immediate and delayed allergic reactions to Crotalidae polyvalent immune Fab (ovine) antivenom. Ann Emerg Med (2002) 39(6):671-6. doi: 10.1067/mem.2002.123134

17. de Silva HA, Pathmeswaran A, Ranasinha CD, Jayamanne S, Samarakoon SB, Hittharage A, et al. Low-dose adrenaline, promethazine, and hydrocortisone in the prevention of acute adverse reactions to antivenom following snakebite: a randomised, double-blind, placebo-controlled trial. PLoS Med (2011) 8(5): e1000435. doi: 10.1371/journal.pmed.1000435

18. Lalloo DG, Theakston RDG. Snake antivenoms. J Toxicol Clin Toxicol (2003) 41(3):277-90. doi: 10.1081/clt-120021113. 317.

19. Williams DJ, Jensen SD, Nimorakiotakis B, Müller R, Winkel KD. Antivenom use, premedication and early adverse reactions in the management of snake bites in rural Papua New Guinea. Toxicon (2007) 49(6):780-92. doi: 10.1016/ j.toxicon.2006.11.026

20. Williams DJ, Habib AG, Warrell DA. Clinical studies of the effectiveness and safety of antivenoms. Toxicon (2018) 150:1-10. doi: 10.1016/j.toxicon. 2018.05.001

21. Hasan SMK, Basher A, Molla AA, Sultana NK, Faiz MA. The impact of snake bite on household economy in Bangladesh. Trop Doct (2012) 42(1):41-3. doi: $10.1258 /$ td.2011.110137

22. Hamza M, Idris MA, Maiyaki MB, Lamorde M, Chippaux J-P, Warrell DA, et al. Cost-Effectiveness of Antivenoms for Snakebite Envenoming in 16 Countries in West Africa. PloS Negl Trop Dis (2016) 10(3):e0004568. doi: 10.1371/journal.pntd.0004568 
23. Quintana-Castillo JC, Estrada-Gómez S, Cardona-Arias JA. Economic evaluations of interventions for snakebites: A systematic review. Clinicoecon Outcomes Res (2020) 12:547-54. doi: 10.2147/CEOR.S259426

24. Lavonas EJ, Anderson VE, Gerardo CJ. In reply to Dr. Shah and Dr. Beuhler. Clin Toxicol (2020) 58(3):223-4. doi: 10.1080/15563650.2019.1641607

25. Shah KR, Beuhler MC. Comment on Early administration of fab antivenom resulted in faster limb recovery in copperhead snake envenomation patients. Clin Toxicol (2020) 58(3):222-3. doi: 10.1080/15563650.2019.1620263

26. Habib AG, Brown NI. The snakebite problem and antivenom crisis from a health-economic perspective. Toxicon (2018) 150:115-23. doi: 10.1016/ j.toxicon.2018.05.009

27. Harrison RA, Oluoch GO, Ainsworth S, Alsolaiss J, Bolton F, Arias A-S, et al. Preclinical antivenom-efficacy testing reveals potentially disturbing deficiencies of snakebite treatment capability in East Africa. PLoS Negl Trop Dis (2017) 11(10):e0005969. doi: 10.1371/journal.pntd.0005969

28. Habib AG, Musa BM, Iliyasu G, Hamza M, Kuznik A, Chippaux J-P. Challenges and prospects of snake antivenom supply in sub-Saharan Africa. PLoS Negl Trop Dis (2020) 14(8):e0008374. doi: 10.1371/journal.pntd.0008374

29. Chippaux JP, Kambewasso A. [Snake bites and antivenom availability in the urban community of Niamey, Niger]. Bull Soc Pathol Exot (2002) 95(3):181-3.

30. Borek HA, Rizer J, Ngo A. Fab antivenom controversy continues. Ann Emerg Med (2017) 70(6):928-9. doi: 10.1016/j.annemergmed.2017.08.002

31. Gerardo CJ, Lavonas EJ. In reply. Ann Emerg Med (2017) 70(6):929-30. doi: 10.1016/j.annemergmed.2017.08.003

32. Anderson VE, Gerardo CJ, Rapp-Olsson M, Bush SP, Mullins ME, Greene S, et al. Early administration of Fab antivenom resulted in faster limb recovery in copperhead snake envenomation patients. Clin Toxicol (2019) 57(1):25-30. doi: 10.1080/15563650.2018.1491982

33. Bucaretchi F, Herrera SRF, Hyslop S, Baracat ECE, Vieira RJ. Snakebites by Crotalus durissus ssp in children in Campinas, São Paulo, Brazil. Rev Inst Med Trop Sao Paulo (2002) 44(3):133-8. doi: 10.1590/s0036-46652002000300004

34. Bucaretchi F, Herrera SR, Hyslop S, Baracat EC, Vieira RJ. Snakebites by Bothrops spp in children in Campinas, São Paulo, Brazil. Rev Inst Med Trop Sao Paulo (2001) 43(6):329-33. doi: 10.1590/s0036-46652001000600006

35. Otero R, Gutiérrez J, Beatriz Mesa M, Duque E, Rodríguez O, Luis Arango J, et al. Complications of Bothrops, Porthidium, and Bothriechis snakebites in Colombia. A clinical and epidemiological study of 39 cases attended in a university hospital. Toxicon (2002) 40(8):1107-14. doi: 10.1016/s0041-0101 (02)00104-6

36. Pinho FMO, Zanetta DMT, Burdmann EA. Acute renal failure after Crotalus durissus snakebite: a prospective survey on 100 patients. Kidney Int (2005) 67 (2):659-67. doi: 10.1111/j.1523-1755.2005.67122.x

37. Nicoleti AF, de Medeiros CR, Duarte MR, França FO de S. Comparison of Bothropoides jararaca bites with and without envenoming treated at the Vital Brazil Hospital of the Butantan Institute, State of São Paulo, Brazil. Rev Soc Bras Med Trop (2010) 43(6):657-61. doi: 10.1590/s0037-86822010000600011

38. Ribeiro LA, Jorge MT, Lebrão ML. Prognostic factors for local necrosis in Bothrops jararaca (Brazilian pit viper) bites. Trans R Soc Trop Med Hyg (2001) 95(6):630-4. doi: 10.1016/s0035-9203(01)90101-4

39. Gerardo CJ, Vissoci JRN, Brown MWJ, Bush SP. Coagulation parameters in copperhead compared to other Crotalinae envenomation: secondary analysis of the F(ab')2 versus Fab antivenom trial. Clin Toxicol (2017) 55(2):109-14. doi: 10.1080/15563650.2016.1250275

40. Gerardo CJ, Vissoci JRN, Evans CS, Simel DL, Lavonas EJ. Does this patient have a severe snake envenomation?: the rational clinical examination systematic review. JAMA Surg (2019) 154(4):346-54. doi: 10.1001/ jamasurg.2018.5069

41. Kanaan NC, Ray J, Stewart M, Russell KW, Fuller M, Bush SP, et al. Wilderness medical society practice guidelines for the treatment of pitviper envenomations in the united states and canada. Wilderness Environ Med (2015) 26(4):472-87. doi: 10.1016/j.wem.2015.05.007

42. Gold BS, Dart RC, Barish RA. Bites of venomous snakes. N Engl J Med (2002) 347(5):347-56. doi: 10.1056/NEJMra013477

43. Thomas L, Tyburn B, Ketterlé J, Biao T, Mehdaoui H, Moravie V, et al. Prognostic significance of clinical grading of patients envenomed by Bothrops lanceolatus in Martinique. Members of the Research Group on Snake Bite in Martinique. Trans R Soc Trop Med Hyg (1998) 92(5):542-5. doi: 10.1016/ s0035-9203(98)90907-5
44. Ward KN, Wortley AG, Quackenbush EB, Gerardo CJ. 396 variability in antivenom treatment in snake envenomations between two major tertiary care emergency departments. Ann Emerg Med (2014) 64(4):S141. doi: 10.1016/ j.annemergmed.2014.07.424

45. Rokyta DR, Wray KP, McGivern JJ, Margres MJ. The transcriptomic and proteomic basis for the evolution of a novel venom phenotype within the Timber Rattlesnake (Crotalus horridus). Toxicon (2015) 98:34-48. doi: 10.1016/j.toxicon.2015.02.015

46. Lomonte B, Tsai W-C, Ureña-Diaz JM, Sanz L, Mora-Obando D, Sánchez EE, et al. Venomics of New World pit vipers: genus-wide comparisons of venom proteomes across Agkistrodon. J Proteomics (2014) 96:103-16. doi: 10.1016/ j.jprot.2013.10.036

47. Bickler PE. Amplification of snake venom toxicity by endogenous signaling pathways. Toxins (Basel) (2020) 12(2). doi: 10.3390/toxins12020068

48. Ibiapina HNS, Costa AG, Sachett JAG, Silva IM, Tarragô AM, Neves JCF, et al. An Immunological Stairway to Severe Tissue Complication Assembly in Bothrops atrox Snakebites. Front Immunol (2019) 10:1882. doi: 10.3389/ fimmu.2019.01882

49. Rex CJ, Mackessy SP. Venom composition of adult Western Diamondback Rattlesnakes (Crotalus atrox) maintained under controlled diet and environmental conditions shows only minor changes. Toxicon (2019) 164:51-60. doi: 10.1016/j.toxicon.2019.03.027

50. Liu Y, Zhang X-H, Yu Y, Chen H-X, Zhou Y-L, Zhang X-X. Snake venom characteristic peptides: novel fingerprints for species identification by sheathless capillary electrophoresis-electrospray ionization-mass spectrometry. Analyst (2020) 145(14):5027-31. doi: 10.1039/d0an00461h

51. Lynch HE, Sanchez AM, D’Souza MP, Rountree W, Denny TN, Kalos M, et al. Development and implementation of a proficiency testing program for Luminex bead-based cytokine assays. J Immunol Methods (2014) 409:62-71. doi: 10.1016/j.jim.2014.04.011

52. Patro ARK, Mohanty S, Prusty BK, Singh DK, Gaikwad S, Saswat T, et al. Cytokine Signature Associated with Disease Severity in Dengue. Viruses (2019) 11(1). doi: 10.3390/v11010034

53. Muema DM, Akilimali NA, Ndumnego OC, Rasehlo SS, Durgiah R, Ojwach DBA, et al. Association between the cytokine storm, immune cell dynamics, and viral replicative capacity in hyperacute HIV infection. BMC Med (2020) 18(1):81. doi: 10.1186/s12916-020-01529-6

54. Kojima N, Siebert JC, Maecker H, Rosenberg-Hasson Y, Leon SR, Vargas SK, et al. Cytokine expression in Treponema pallidum infection. J Transl Med (2019) 17(1):196. doi: 10.1186/s12967-019-1947-7

55. White RR, Weber RA. Poisonous snakebite in central Texas. Possible indicators for antivenin treatment. Ann Surg (1991) 213(5):466-71. doi: 10.1097/00000658-199105000-00012

56. Seifert SA, Kirschner R I, Martin N. Recurrent, persistent, or late, new-onset hematologic abnormalities in Crotaline snakebite. Clin Toxicol (2011) 49 (4):324-9. doi: 10.3109/15563650.2011.566883

57. Belard A, Buchman T, Dente CJ, Potter BK, Kirk A, Elster E. The uniformed services university's surgical critical care initiative (sc2i): bringing precision medicine to the critically ill. Mil Med (2018) 183(suppl_1):487-95. doi: 10.1093/milmed/usx164

58. Gelbard RB, Hensman H, Schobel S, Khatri V, Tracy BM, Dente CJ, et al. Random forest modeling can predict infectious complications following trauma laparotomy. J Trauma Acute Care Surg (2019) 87(5):1125-32. doi: 10.1097/TA.0000000000002486

59. Bradley M, Dente C, Khatri V, Schobel S, Lisboa F, Shi A, et al. Advanced modeling to predict pneumonia in combat trauma patients. World J Surg (2020) 44(7):2255-62. doi: 10.1007/s00268-019-05294-3

60. Dente CJ, Bradley M, Schobel S, Gaucher B, Buchman T, Kirk AD, et al. Towards precision medicine: Accurate predictive modeling of infectious complications in combat casualties. J Trauma Acute Care Surg (2017) 83 (4):609-16. doi: 10.1097/TA.0000000000001596

61. Lisboa FA, Bradley MJ, Hueman MT, Schobel SA, Gaucher BJ, Styrmisdottir EL, et al. Nonsteroidal anti-inflammatory drugs may affect cytokine response and benefit healing of combat-related extremity wounds. Surgery (2017) 161 (4):1164-73. doi: 10.1016/j.surg.2016.10.011

62. Vissoci JRN, Tupetz A, Phillips AJ, Kelly PE, Lavonas EJ, Gerardo CJ. What activities do snakebite envenomation patients choose to assess dysfunction? Toxicon (2020) 182:S16-7. doi: 10.1016/j.toxicon.2020.04.043 
63. Gutiérrez JM, Burnouf T, Harrison RA, Calvete JJ, Brown N, Jensen SD, et al. A Call for Incorporating Social Research in the Global Struggle against Snakebite. PLoS Negl Trop Dis (2015) 9(9):e0003960. doi: 10.1371/ journal.pntd.0003960

64. Larson BA. Calculating disability-adjusted-life-years lost (DALYs) in discretetime. Cost Eff Resour Alloc (2013) 11(1):18. doi: 10.1186/1478-7547-11-18

65. Júnior FAN, Jorge ARC, Marinho AD, Silveira JA de M, Alves NTQ, Costa PHS, et al. Bothrops alternatus Snake Venom Induces Cytokine Expression and Oxidative Stress on Renal Function. Curr Top Med Chem (2019) 19 (22):2058-68. doi: 10.2174/1568026619666190809100319

66. Stone SF, Isbister GK, Shahmy S, Mohamed F, Abeysinghe C, Karunathilake H, et al. Immune response to snake envenoming and treatment with antivenom; complement activation, cytokine production and mast cell degranulation. PLoS Negl Trop Dis (2013) 7(7):e2326. doi: 10.1371/journal.pntd.0002326

67. Yin S, Kokko J, Lavonas E, Mlynarchek S, Bogdan G, Schaeffer T. Factors associated with difficulty achieving initial control with crotalidae polyvalent immune fab antivenom in snakebite patients. Acad Emerg Med (2011) 18 (1):46-52. doi: 10.1111/j.1553-2712.2010.00958.x

68. Freiermuth CE, Lavonas EJ, Anderson VE, Kleinschmidt KC, Sharma K, Rapp-Olsson M, et al. Antivenom Treatment Is Associated with Fewer Patients using Opioids after Copperhead Envenomation. West J Emerg Med (2019) 20(3):497-505. doi: 10.5811/westjem.2019.3.42693

69. Seifert SA, Mascarenas DN, Fullerton L, Warrick BJ, Smolinske SC. Unpredicted late-, new-onset thrombocytopenia and hypofibrinogenemia in Fab antivenom-treated rattlesnake envenomation. Toxicon (2020) 184:55-6. doi: 10.1016/j.toxicon.2020.05.026

70. Lavonas EJ, Gerardo CJ. Letter to the editor: "Safety of nonsteroidal antiinflammatory drugs in copperhead snakebite patients" by Pham and Mullins, Clin Toxicol 2018 May 18. Clin Toxicol (2019) 57(2):144-5. doi: 10.1080/ 15563650.2018.1502445

71. Pham HX, Mullins ME. Safety of nonsteroidal anti-inflammatory drugs in copperhead snakebite patients. Clin Toxicol (2018) 56(11):1121-7. doi: $10.1080 / 15563650.2018 .1468447$
72. Cezarette GN, Sartim MA, Sampaio SV. Inflammation and coagulation crosstalk induced by BJcuL, a galactose-binding lectin isolated from Bothrops jararacussu snake venom. Int J Biol Macromol (2020) 144:296304. doi: 10.1016/j.ijbiomac.2019.12.015

73. de Almeida MT, Freitas-de-Sousa LA, Colombini M, Gimenes SNC, Kitano ES, Faquim-Mauro EL, et al. Inflammatory Reaction Induced by Two Metalloproteinases Isolated from Bothrops atrox Venom and by Fragments Generated from the Hydrolysis of Basement Membrane Components. Toxins (Basel) (2020) 12(2). doi: 10.3390/ toxins 12020096

74. Wellmann IAM, Ibiapina HNS, Sachett JAG, Sartim MA, Silva IM, Oliveira SS, et al. Correlating Fibrinogen Consumption and Profiles of Inflammatory Molecules in Human Envenomation's by Bothrops atrox in the Brazilian Amazon. Front Immunol (2020) 11:1874. doi: 10.3389/ fimmu.2020.01874

75. Gerardo CJ, Evans CS, Kuchibhatla M, Mando-Vandrick J, Drake WG, Yen M, et al. Time to antivenom administration is not associated with total antivenom dose administered in a copperhead-predominant snakebite population. Acad Emerg Med (2015) 22(3):308-14. doi: 10.1111/acem.12598

Conflict of Interest: ES was employed by DecisionQ. CG receives grant funding from BTG Specialty Pharmaceuticals.

The remaining authors declare that the research was conducted in the absence of any commercial or financial relationships that could be construed as a potential conflict of interest.

Copyright $(02021$ Gerardo, Silvius, Schobel, Eppensteiner, McGowan, Elster, Kirk and Limkakeng. This is an open-access article distributed under the terms of the Creative Commons Attribution License (CC BY). The use, distribution or reproduction in other forums is permitted, provided the original author(s) and the copyright owner(s) are credited and that the original publication in this journal is cited, in accordance with accepted academic practice. No use, distribution or reproduction is permitted which does not comply with these terms. 